\title{
Event-Related Analysis for Event Types of Fixed Order and Restricted Spacing by Temporal Quantification of Trial-Averaged fMRI Time Courses
}

\author{
Hannes Ruge, MSc, ${ }^{*}$ Marcel Brass, PhD, Gabriele Lohmann, PhD, and \\ D. Yves von Cramon, MD
}

\begin{abstract}
Purpose: To develop a method for event-related fMRI that allows rapidly presented event sequences to be analyzed, without requiring transitions of different event-types to be counterbalanced.
\end{abstract}

Materials and Methods: A cued task switching procedure was investigated with an experimental trial comprising a visual task cue that indicated how to process a subsequent visual target stimulus. Cue and target were either presented quasi-simultaneously, separated by a 100 msec cue-targetinterval (CTI100), or the target presentation was delayed by $2000 \mathrm{msec}$ (CTI2000). To characterize the trial-related BOLDresponse in terms of its temporal relation to the underlying event structure, the pattern of onset latency differences and peak latency differences for CTI2000 minus CTI100 was evaluated. Independent estimates of onset latencies and peak latencies were determined for preprocessed trial-averaged time courses by jackknife resampling.

Results: Validating results were obtained for two brain areas with known characteristics: the visual cortex (cue-locked plus target-locked activation) and the motor cortex (responselocked activation). Extending the analysis to prefrontal areas with a priori unknown characteristics differentiated between several meaningful temporal activation patterns.

Conclusion: The method yielded a fine-grained temporal description of trial-related BOLD-responses that could be successfully used for the event-related analysis of an experimental design that was highly restricted with respect to event order and event spacing.

Key Words: event-related fMRI; rapid stimulation; fixed event order; restricted event spacing; temporal BOLD-response characterization; jackknife resampling.

J. Magn. Reson. Imaging 2003;18:599-607.

(c) 2003 Wiley-Liss, Inc.

IN RECENT YEARS, event-related functional MRI (fMRI) has become an established neuroimaging technique

Max-Planck-Institute of Cognitive Neuroscience, Leipzig, Germany. Contract grant sponsor: German-Israeli Foundation for Scientific Research and Development (GIF); Contract grant number: G-635-88.4/1999.

*Address reprint requests to: H.R., Max-Planck-Institute of Cognitive Neuroscience, Stephanstr. 1a, 04103 Leipzig, Germany. E-mail: ruge@cns.mpg.de Received March 7, 2003; Accepted July 15, 2003.

DOI 10.1002/jmri.10397

Published online in Wiley InterScience (www.interscience.wiley.com).
$(1,2)$. In contrast to traditional blocked designs, it has become possible to investigate more natural and flexible experimental designs. The obvious advantage of analyzing blood-oxygenation-level-dependent (BOLD) responses to single events is that different event types can be presented in random sequence, hence avoiding typical confounds related to blocked designs (3). On the one hand, realizing randomized event sequences meets the demands of a wide range of experimental settings. On the other hand, common techniques for the analysis of event-related fMRI designs necessarily require randomization to obtain counterbalanced transition frequencies between different event types. This is a prerequisite to compensate signal overlap between BOLD responses associated with successive events $(4,5)$. This holds especially for experimental designs with fixed and/or short spacing between events. Consequently, when attempting to realize experimental designs in which, for logical reasons, event types cannot be counterbalanced and in which the free choice of arbitrary event spacings are additionally restricted, considerable problems arise with respect to event-related analysis.

This article proposes an approach that overcomes some constraints of existing methods to analyze event sequences with fixed order and restricted event spacing choice. The introduction gives a short overview of relevant available methods, then describes the concrete experimental design to be investigated, and ends with a sketch of the proposed method.

A growing literature has been concerned with the optimization of event-related fMRI designs (4-6). The most relevant aspect to be considered refers to the intrinsic problem that hemodynamic changes reflected by the measured BOLD response do not provide a realtime indicator of the underlying neural activity associated with distinct events. The BOLD impulse response elicited by short single events is blurred, extending over 10-15 seconds (1). This implies a strong overlap of BOLD responses associated with rapidly presented events. The most conservative procedure to deal with BOLD overlap is to separate single events in the range of the extent of a single-event impulse BOLD response (7). However, if appropriate pseudorandomization of event order and event spacing is considered, compo- 
nent BOLD responses can be disentangled even for very narrow event spacings of seconds and below (4-6,810). Counterbalancing transitions between different event-types guarantees that the past and future time course enclosing the present event can be simply subtracted out. In this case, the selective averaging framework (8) or techniques based on the general linear model (11) can be successfully used to determine the contribution of the signal of interest.

If the event ordering is fixed due to experimental constraints the variation of event spacings still allows the BOLD components associated with distinct events to be disentangled, given that the range of varied event spacings is comparably large, e.g., a variation between one and 12 seconds (12).

Deconvolution techniques as proposed by Glover (13) that can be used to recover the characteristics of superimposed BOLD impulse responses, seem to face a lower limit for event spacing of approximately four seconds (13). A similar limit was evident for explicit parameterization of temporal features of overlapping BOLD responses (14).

While the methods sketched above allow for flexibility in developing event-related designs that meet the demands of many potentially interesting experimental settings, there are still some important constraints. In particular, this holds for experimental settings where the randomization of event order and event spacings is restricted, as is the case in cueing procedures. Here, event order is logically restricted to first presenting the cue and then presenting the target stimulus. Furthermore, the cue-target interval (CTI) might be required to be fixed (for better control over preparation onset) and/or comparably short (to avoid intervening uncontrolled cognition).

It is exactly these issues that are relevant in the cued task-switching procedure $(15,16)$ we investigated. The stimulation protocol consists of pseudorandomized trial types, spaced by six seconds. Trial types are defined according to a two-by-two design, with the independent variables task-transition (task switch vs. task repeat) and CTI (100 msec vs. $2000 \mathrm{msec}$ ). Within a trial, two distinct event types are realized: task cue and target stimulus. The order of event types cannot be counterbalanced, because an experimental trial must logically comprise both event types in fixed order (first cue, then target). Moreover, the cue-target interval (CTI) must alternate between two fixed values-one very short interval (100 msec) to maximize switch demands, and a sufficiently long (but not too long) interval (2000 msec) to relax switch demands without introducing an additional working memory component. For a more elaborate conceptual background see Meiran (15). Similar experimental demands are expected to arise in many other "higher cognition" paradigms in which a trial consists of more than one single event, e.g., priming experiments, memory retrieval experiments, etc.

In order to analytically access this highly restricted design in an event-related fashion, we propose a procedure that relies on the independent quantification of onset latencies and peak latencies of trial-averaged BOLD responses. Latencies of the BOLD response at CTI100 are used as a reference; they are sufficiently invariant in spite of exhibiting a purely cue-locked, a purely target-locked, or a combined cue-locked plus target-locked activation. In contrast, latencies of the trial-related BOLD response at CTI2000 vary depending on the specific type of event-locking. Distinct patterns of latency differences between CTI100 and CTI2000 yield yes-no decisions on the presence or absence of BOLD components associated with different event types.

The basic validity of the proposed method is demonstrated for two brain areas with known characteristics: the extrastriate visual cortex and the motor cortex. While the visual cortex is supposed to exhibit BOLD activation for both visual cues and visual targets, the motor cortex is supposed to be time-locked activated exclusively to target presentation (which is invariably locked to the motor response). Due to the specific criteria we used for the identification of BOLD onsets and peaks, this validation should provide clues about the robustness of the analysis with respect to nonlinear effects of BOLD superposition and possible biases. Additionally, we applied the same analysis to three exemplary brain areas in the frontal cortex with unknown BOLD signal temporal characteristics, which we found to be involved in task-switching-related processes.

\section{MATERIALS AND METHODS Subjects}

We measured 22 subjects who all gave written informed consent to participate in the present study. No subject had a history of neurological disorder, major medical disorder, or psychiatric disorder. All subjects were right-handed as assessed by the Edinburgh Inventory (17). Four subjects were excluded due to movement artifacts. Mean age was 25.5 years (range $21-35$ years), and 10 were female.

\section{Experimental Procedure}

We used a spatial task switching procedure introduced by Meiran (15) and Meiran et al (16). A randomly-varying task cue defined the rules that specified how to process the subsequent target stimulus. The task cue was displayed either $100 \mathrm{msec}$ (cue-target-interval CTI100) or 2000 msec (cue-target-interval CTI2000) before presentation of a target stimulus. The manipulation of CTI was intended to compare task switching effects given the opportunity to prepare in advance (CTI2000) or not (CTI100). One task (left-right judgment) required subjects to indicate the position of a small square within a two-by-two grid on the horizontal dimension. Analogously, the alternative task (up-down judgment) required subjects to indicate the square's position in the vertical dimension. Two response buttons located left-down (left index finger) and right-up (right index finger) were used for indicating left or right in one task and up or down in the alternative task. A two-by-two experimental design with independent variables task transition (task switch vs. task repeat) and preparation interval (CTI100 vs. CTI2000) was introduced. Conditions were pseudorandomized to obtain approximately balanced single-condition frequencies 


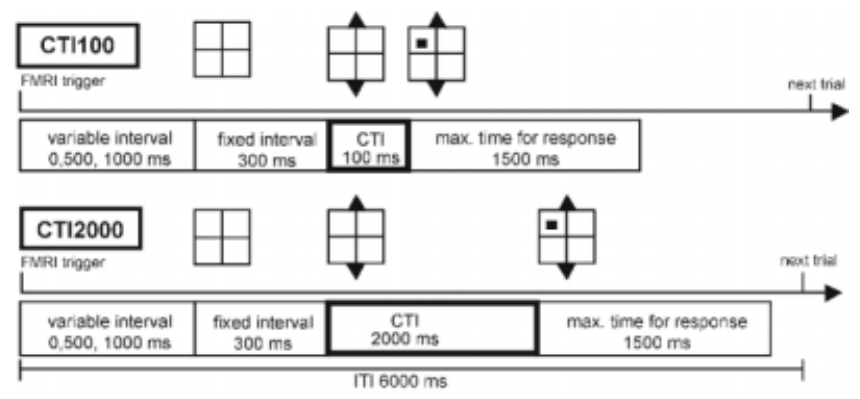

Figure 1. Timing for the cue-target interval of $100 \mathrm{msec}$ (CTI100) and for the cue-target interval of $2000 \mathrm{msec}$ (CTI2000). In the left-right task, subjects had to decide if the small square appeared on the left or on the right part of the grid. The up-down task required subjects to indicate the position of the target on the vertical dimension. The task subjects had to perform was indicated by arrow cues at the edge of the grid.

and balanced transition frequencies. For each of the four conditions, on average, 32 trials were done for each subject. The individual trials were triggered every six seconds by the MRI control. To improve the temporal resolution, we introduced three different oversampling intervals $(0,500$, and $1000 \mathrm{msec})$ at the beginning of each trial (11). This implied a temporal resolution of $500 \mathrm{msec}$, given a TR $=1.5$ seconds. We furthermore randomly interspersed a total of 16 null event trials. The exact timing of the experimental procedure is described in Figure 1.

\section{MRI Procedure}

The experiment was carried out on a $3 \mathrm{~T}$ scanner (Medspec 30/100, Bruker, Ettlingen, Germany). Sixteen axial slices (19.2 cm FOV, 64 by 64 matrix, $5 \mathrm{~mm}$ thickness, $2 \mathrm{~mm}$ spacing) were acquired parallel to the AC-PC plane and covering the whole brain. We used a single shot, gradient-recalled echo planar imaging (EPI) sequence (time-to-repeat $1500 \mathrm{msec}$, echo-time 30 msec, $90^{\circ}$ flip angle). Prior to the functional runs, corresponding sets of 16 anatomical modified driven equilibrium Fourier transform (MDEFT)-slices and 16 EPI-T1 slices were acquired. Stimuli were displayed on a back-projection screen, mounted in the bore of the magnet behind the participant's head, by using a liquid crystal display (LCD) projector. Participants viewed the screen behind them by wearing mirrored glasses.

\section{Preprocessing}

The preprocessing of whole-brain fMRI data was performed by using the LIPSIA software package (18). To align the functional data slices with the Talairach threedimensional stereotactic coordinate reference system (19), a rigid linear registration with six degrees of freedom (three rotational and three translational) was performed. The rotational and translational parameters were obtained on the basis of the MDEFT and EPI-T1 slices. The parameters were subsequently transformed to standard Talairach brain size by linear scaling. The resulting parameters were then used to transform the functional slices by using trilinear interpolation, so that the resulting functional slices were aligned with the stereotactic coordinate system. The functional data were first corrected for movement artifacts. Further, the temporal offset between the slices acquired in one scan was corrected by using a sinc-interpolation algorithm. Data were smoothed using a spatial Gaussian filter with full width half maximum (FWHM) $=5.7 \mathrm{~mm}$. A temporal high pass filter with a mean cutoff frequency of $1 / 173 \mathrm{~Hz}$ (range $1 / 122-1 / 238 \mathrm{~Hz}$ ) was applied for baseline correction. Considering the design frequency, the cutoff-frequency was determined for each subject individually, according to the maximal pairwise temporal distance of trials for the experimental conditions, with this distance being minimal. The temporal analysis of trial-averaged time courses was based on these preprocessed data.

\section{Defining Regions of Interest}

Regions of interest (ROIs) for the extraction of trialaveraged time courses were generated based on the general linear model for serially autocorrelated observations (20). The design matrix for event-related analysis was created by using a model of the hemodynamic response, with a variable delay (21). Event onsets were time-locked to the task cue. ${ }^{1}$ The model equation, including the observation data, the design matrix, and the error term, was convolved with a Gaussian kernel with a dispersion of four seconds FWHM. The increased autocorrelation due to filtering was taken into account during statistical evaluation. Contrast maps were generated for each subject. A one-sample $t$-test of contrast maps across subjects (random effects model) was computed to determine if observed differences between conditions were significantly different from zero. Subsequently, $\mathrm{t}$ values were transformed into $\mathrm{z}$ scores.

For validation purposes, we analyzed the right extrastriate visual cortex ( $\mathrm{VC}$ ) and the right motor cortex (MC). The VC was identified by contrasting all combined experimental conditions against the null event condition. The MC was identified by contrasting left hand finger presses against right hand finger presses. To apply our method to brain areas with unknown temporal characteristics, we selected three exemplary frontal regions that showed stronger activation in switch trials than in repeat trials. In total, we selected five ROIs for the analysis of trial-averaged time courses (see Table 1).

\section{Analysis of Trial-Averaged Time Courses}

The aim was to map the temporal within-trial event structure onto temporal characteristics (onset latencies and peak latencies) of the trial-related BOLD response, to determine which event induced the observed BOLD response. Figure 2 schematically depicts prototypical trial-averaged time courses resulting from three different combinations of two components BOLD response each being associated with one or two events occurring

${ }^{1}$ We obtained highly similar results irrespective of either cue-locked or target-locked analysis and irrespective of including two or three basis functions. 
Table 1

Exact Results of the Jackknife Analysis for Onset Latency Differences (CTI2000-CTI100) and Peak Latency Differences (CTI2000 - CTI100)

\begin{tabular}{lcrr}
\hline \multicolumn{1}{c}{ Region of Interest } & Talairach & Zmax & Dif. onsets \\
\hline Right extrastriate visual cortex (VC) & $13-922$ & 6.02 & $-0.09 \pm 0.12$ \\
Right motor cortex (MC) & $33-2447$ & 3.99 & $2.21 \pm 0.21$ \\
Left posterior inferior frontal sulcus (plFS) & -41630 & 3.81 & $-0.08 \pm 0.22$ \\
Pre-supplementary motor area (PreSMA) & 21350 & 3.31 & $0.87 \pm 0.36$ \\
Anterior frontomedian cortex (aFMC) & 42338 & 5.24 & $1.40 \pm 0.13$ \\
\hline
\end{tabular}

Mean \pm SE.

at discrete time points: the task cue and the target stimulus. The cue-related component curve (solid black) and the null event curve (dotted black, Fig. 2c) were derived from real-data trial-averages that were subsequently modified (shifted and strongly smoothed). These curves are used for idealized demonstration purposes but do not reflect an empirically valid signal time course. The target-related component curve (dotted gray) was derived from the cue-related component curve by a shift of two seconds. The cue-related plus target related curve (dashed gray) was obtained by linear- and equally-weighted superposition of both single components. The curves in Figure 2c (with intertrial overlap of the BOLD signal) were obtained by adding a prototypical null event time course (dotted black) to each of the three model curves shown in Figure 2b (without intertrial overlap).

The time course at CTI100 (Fig. 2a) provides a reference that is assumed to be sufficiently invariant with respect to its onset latencies and peak latencies, irrespective of being cue-related, target-related, or both. In contrast, onset latencies and peak latencies of the BOLD response for CTI2000 (Fig. 2b) do vary depending on differential contributions of a cue component and a target component. Hence, considering distinct patterns of latency differences between CTI100 and CTI2000 provides information about the specific event-locking of a trial-related BOLD response at CTI2000.

\section{Onset Latencies}

Differences in onset latencies yield precise information on the point in time when neural activity starts during the 2000 msec CTI (bounded by the task cue at time point 0 msec and the target stimulus at time point 2000 msec). The two landmark patterns depicted in Figure 2b (cue-locked and purely target-locked) represent the extrema of a continuum between $0 \mathrm{msec}$ and $2000 \mathrm{msec}$. Any internally-induced event occurring at a point in time during the $2000 \mathrm{msec}$ CTI would be reflected by differences in onset latencies greater than $0 \mathrm{msec}$ and smaller than 2000 msec.

\section{Peak Latencies}

Differences in peak latencies provide additional information to determine if a first BOLD component timelocked to a given event 1 is followed by a second component time-locked to a given event 2 . If the peak latency difference equals the onset latency difference, the BOLD response at CTI2000 reflects an activation exclusively related to event 1 . If the peak latency difference exceeds the onset latency difference, one can infer that event 1 is followed by a second event. Unfortunately, there is no way to infer the relative temporal distance between event 1 and event 2 from the observed peak latency difference, since this value is also modulated by the relative amplitude strengths of the superimposed BOLD components.

The following list gives an overview of possible inferences one can draw from distinct patterns of latency differences resulting from the discrete cue-target event structure described in Figure 2. Importantly, these three patterns represent the landmark examples of a continuum of temporal event structures that are possible within the basic cue-target design (e.g., internallyinduced events occurring during the 2000 msec CTI or sustained activity that might be approximated by a cascade of subsequent events during the $2000 \mathrm{msec}$ CTI). The variety of intermediate patterns is bounded by these three landmark patterns.

- A purely cue-locked activation is characterized by: 1) equal onset latencies, and 2) equal peak latencies for CTI100 and CTI2000.

- A purely target-locked activation is characterized by: 1) shifted onset latency, and 2) shifted peak latency for CTI2000 compared to CTI100 (about two seconds).

- A combined cue-locked plus target-locked activation is characterized by: 1) equal onset latencies for both CTIs, and 2) shifted peak latency for CTI2000 compared to CTIIOO.

The schematic model was based on the assumption of linear BOLD superposition, although we were aware that nonlinear effects exist $(8,13,22)$. Nonlinearities increase with shorter event spacing, resulting in reduced amplitudes and prolonged peak latencies $(23,24)$. Importantly, this does not hold for onset latencies that seem to be unaffected by nonlinear effects. The analysis of the visual cortex and the motor cortex is intended to estimate distortions due to nonlinearity.

Since we always compute relative onset latency differences and peak latency differences within regions, we can compensate for variations in the shape of the BOLD response specific to different brain areas. This is an important prerequisite, since it is known that temporal characteristics of the BOLD response may differ significantly between brain areas $(25,26)$.

Figure $2 \mathrm{~b}$ shows the three potential landmark BOLD responses at CTI2000 that we proposed above, without 


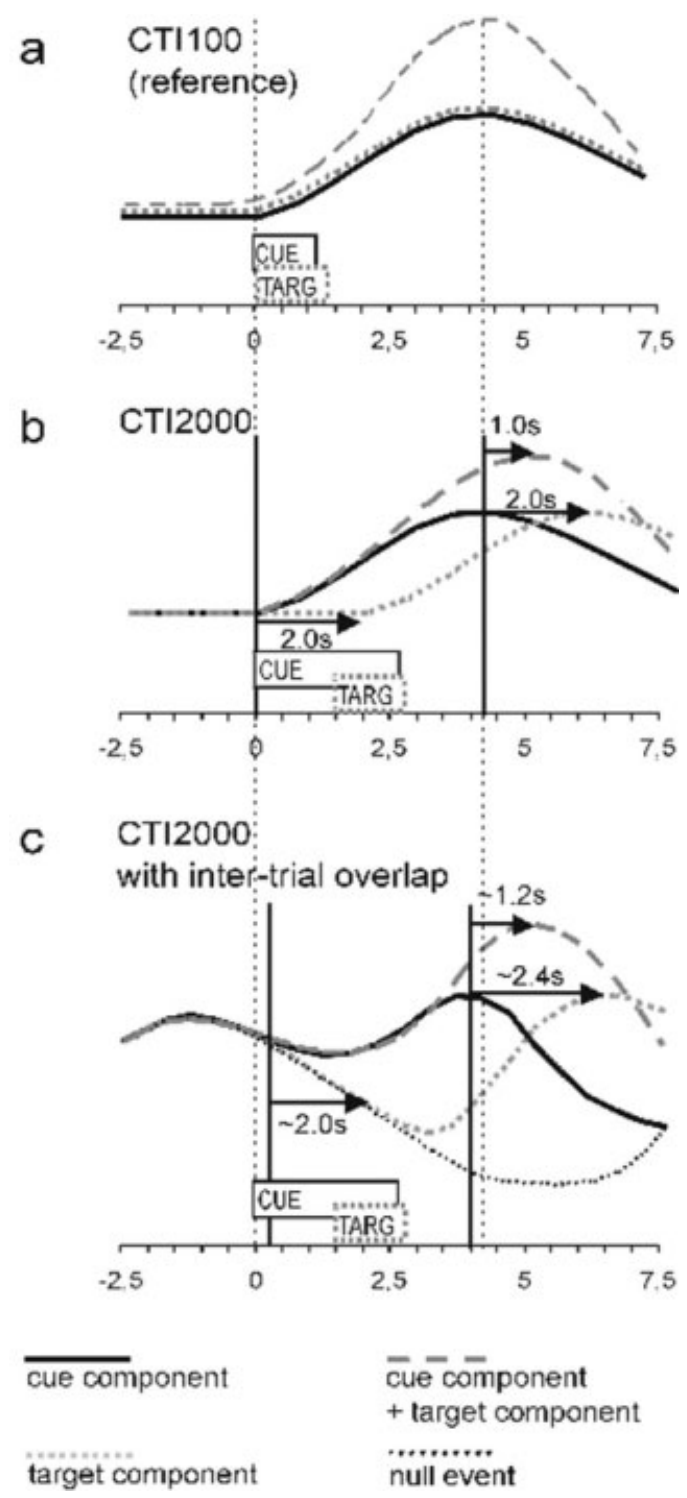

Figure 2. All three graphs schematically depict different prototypical courses of BOLD activation depending on the differential contribution of a cue-related BOLD component and a target-related BOLD component. The trial shown starts at time point zero. Three landmark patterns are suggested: 1) purely cue-locked activation (solid black); 2) purely target-locked activation (dotted gray); 3) combined cue-locked plus targetlocked activation (dashed gray). a: Cue-target-interval $=100$ msec (CTI100). b: Cue-target-interval $=2000 \mathrm{msec}$ (CTI2000). The cue-locked component at CTI2000 is assumed to be identical with each of the separate components at CTI100. c: Same as (b) but considering intertrial overlap of the BOLD signal. The black dotted line represents the null-event time course. The arrows illustrate the shift of both the onset latencies and the peak latencies at CTI2000, referenced to the corresponding latencies at CTI100. In time courses with intertrial BOLD overlap, BOLD onsets are determined via the inflection point of the curve.

intertrial overlap of the BOLD signal. Since individual trials were spaced by six seconds, a strong intertrial BOLD overlap is to be expected. Figure $2 \mathrm{c}$ incorporates this intertrial overlap. As shown schematically, the relevant latency differences may be subject to some dis- tortion (this holds in particular with respect to peak latency differences).

Moreover, the onset of trial-related BOLD responses is hidden within the decreasing flank of the BOLD response associated with the previous trial. Two compensatory strategies were considered. First, the intertrial overlap can be eliminated by subtracting the trial-averaged time course of null events from those associated with the experimental conditions (4). Null events are periods without stimulation or tasks to be performed by the subject. Hence, the BOLD signal is assumed to decay passively. Given that transitions between null event trials and experimental trials are counterbalanced, subtracting null events should filter out the overlap from preceding and subsequent trials. However, this logic is idealized since it is known that many brain areas also show BOLD activation in null event trials or during comparable resting baselines (27). A more general approach is to determine the onset directly within the decreasing flank of the preceding BOLD response. The inflection point within this flank is supposed to be the most adequate landmark, since it is as close as possible to the original onset time. According to results from Bandettini and Cox (7) an inter trial interval (ITI) of six seconds is expected to be quite adequate to produce a pronounced decreasing flank, hence providing a sufficiently wide range of inflection point variability.

The results obtained by null event subtraction were quite discouraging, in particular with respect to BOLD onsets. Therefore, the present analyses focus exclusively on the inflection point approach for extracting onset latencies.

To obtain the inflection point, the interpolated accurate time-point when the second derivative intersects zero was computed. The search range was restricted to a time-window from zero seconds to four seconds, relative to trial onset.

Reliably determining onsets and peaks in single-subject data turned out to be difficult. Therefore, singlesubject data were further averaged across subjects (grand-average). This additional averaging process allowed us to extract unique values for onsets and peaks more reliably, while any information about the variability of the extracted values was lost. The jackknife resampling procedure $(28,29)$ provides an elegant tool to create a statistical distribution from grand-averaged values. Each of $N$ subjects is excluded from grandaveraging once. The resulting distribution of $N$ grandaverages (each omitting a different subject), can then be used to calculate estimates of standard-errors or other statistics (see Eqs. [1] and [2]). ${ }^{2}$ In research on eventrelated brain potentials (ERP), the jackknife approach has been successfully applied to detect and statistically

${ }^{2}$ Under some circumstances, the jackknife is known to provide less accurate estimates of variability than the more general method of bootstrapping. The purpose of using the jackknife procedure in the present case is to smooth the data by grand-averaging. Thus, including as many trial-averages as possible $(N-1)$ in each sample is supposed to be more important than obtaining better statistical properties. This argument holds especially for error estimates of mean values, which are less vulnerable than, for instance, error estimates of median values or variances. 
evaluate onsets of the lateralized readiness potential (30-32).

The jackknife algorithm for the standard-error of a mean:

$$
s e=\sqrt{\frac{N-1}{N} \sum_{i_{-1}=1}^{N}\left(x i_{-1}-X\right)^{2}}
$$

with $\mathrm{xi}_{-1}$ representing the grand-averages without subject $i$ and $X$ representing the mean of all grand-averages. Similarly, standard errors of mean-differences may be estimated as well:

$$
s e=\sqrt{\frac{N-1}{N} \sum_{i-1=1}^{N}\left(d i_{-1}-D\right)^{2}}
$$

with $\mathrm{di}_{-1}$ representing the grand-average differences without subject $i$ and $D$ representing the mean-difference of all grand-averages.

Interpolation proceeded in two steps. First, we linearly interpolated the preprocessed trial-averaged individual time courses (18) resulting in one point every 125 msec (considering 500 msec oversampling due to variable jitter interval). The jackknifed grand-averages were smoothed (discrete Gaussian approximation considering one adjacent time-point) to compensate for lack of smoothness in the to-be-computed derivatives due to linear interpolation. Derivatives were then obtained by discrete Taylor polynomial approximation. In a second step of interpolation, the accurate time-point of zero intersection was determined for the second derivative of the jackknifed grand-averages. Peak latencies were computed analogously by considering the first derivative within a time-window of 3.5 to 8 seconds.

\section{RESULTS}

Figure 3 and Table 1 depict the results for the VC and the MC that were intended to provide evidence for the basic validity of the method.

\section{Right VC}

As predicted, the VC exhibits a pattern compatible with a combined cue-locked plus target-locked activation, reflected by no significant difference $(0.09 \pm 0.12 \mathrm{sec}-$ onds) in onset latencies (indicating the cue-related BOLD component), and a shifted peak latency of $1.49 \pm$ 0.16 seconds, significantly greater $(P<0.05$, or twice the standard error) than zero seconds (indicating the subsequent target-related BOLD component). The schematic model (Fig. 2) predicted a shifted peak latency of about one second for an equally-weighted summation of a cue component and a target component. The fact that the VC exhibits a shift of 1.49 seconds (significantly different from one second) is assumed to reflect a more pronounced target-locked component relative to the cue-locked component. right VC (13 -92 2)

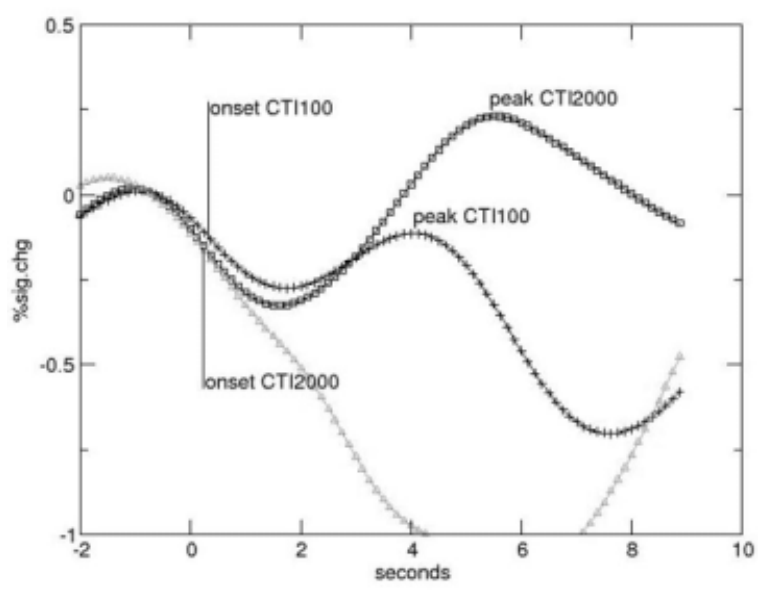

right MC (33-24 47)

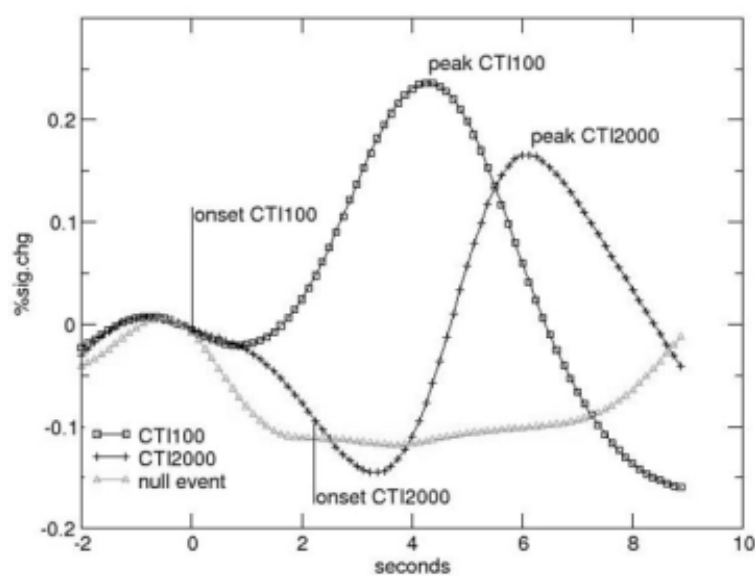

Figure 3. Grand-averages of trial-averaged time courses for the VC and the MC. Black curves marked by squares = CTI100, black curves marked by crosses = CTI2000, gray curves marked by triangles = null event. The temporal resolution of $125 \mathrm{msec}$ was obtained by linear interpolation. The VC exhibits a cue-locked plus target-locked activation (with the same onset latencies for CTI100 and CTI2000, paralleled by a shifted peak latency for CTI2000). The MC exhibits a target/response locked activation (with a shifted onset latency and shifted peak latency of about two seconds for CTI2000).

\section{Right MC}

The MC shows a pattern in line with the predicted target/response-locked activation being reflected by a shifted onset latency of $2.21 \pm 0.21$ seconds (not significantly different from two seconds) and a shifted peak latency of $1.82 \pm 0.11$ seconds (not significantly different from two seconds).

Distortions due to effects of nonlinear BOLD superposition or due to the inflection point criterion are not evident with respect to BOLD onset differences for both the VC and the MC. While the VC is not suitable to assessing distortions with respect to peak latency differences (because of weighting effects induced by the superposition of the cue component and the target component), the results obtained for the MC suggest 
that there is indeed no significant distortion of peak latency differences.

The analysis of three exemplary task-switching-related brain areas, i.e., the posterior inferior frontal sulcus (pIFS), the pre-supplementary motor area (PreSMA), and the anterior fronto-median cortex (aFMC), demonstrate that our method is capable of

left post. IFS (-41 630$)$

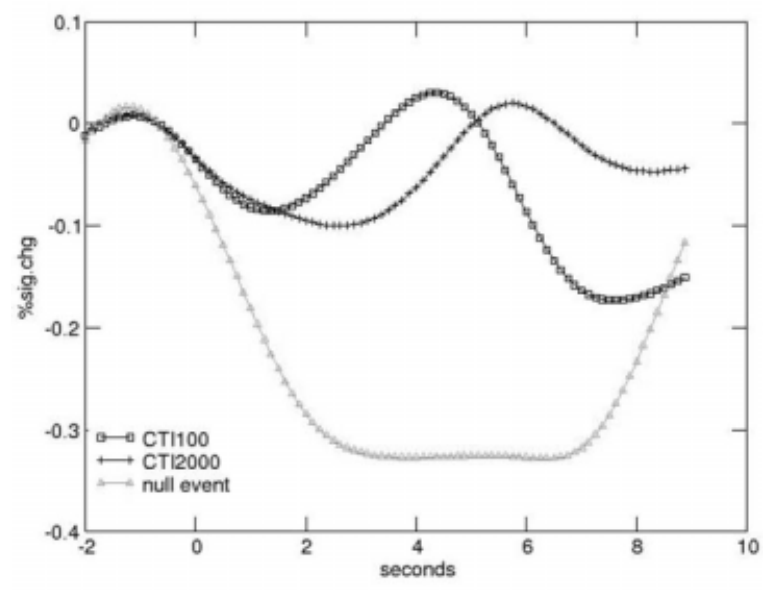

PreSMA (2 13 50)

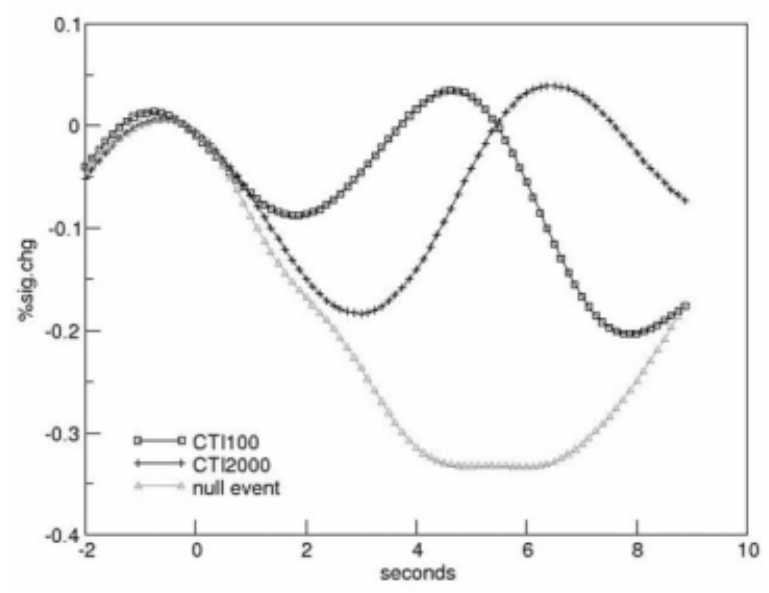

aFMC (4 2338$)$

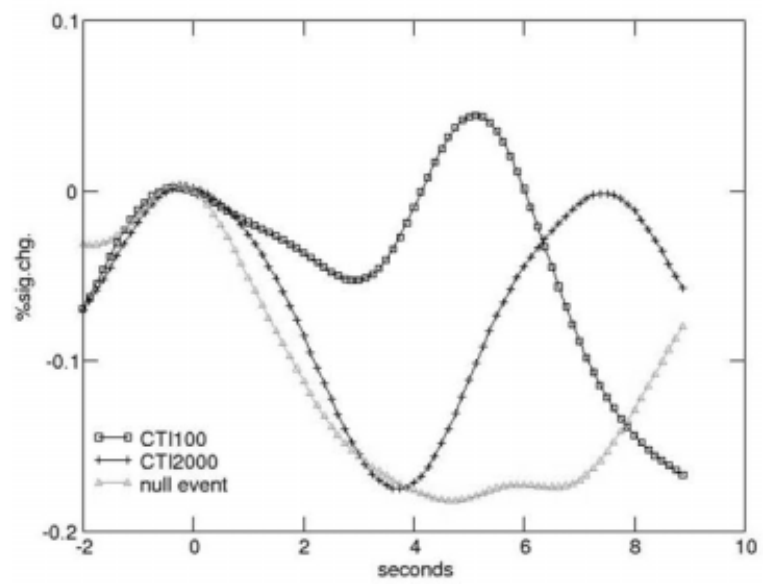

distinguishing between a variety of potentially possible activation patterns (see Fig. 4 and Table 1). While the pIFS is activated cue-locked and target-locked, the aFMC exhibits a purely target-locked pattern. Furthermore, the Pre-SMA gets activated within the two second preparation interval, indicated by a shifted onset latency of $0.87 \pm 0.36$ seconds.

\section{Posterior IFS}

Similar to the VC, the peak latency difference for the pIFS significantly deviates from the expected value of about one second ( $1.40 \pm 0.13$ seconds). In this case, the deviation probably reflects a diminished cue-locked BOLD component relative to the target-locked component. This interpretation is supported by the observation that the dip after the onset is delayed for CTI2000, indicating that the cue component at CTI2000 is diminished as compared to the reference BOLD response at CTI100.

\section{Anterior FMC}

The peak latency difference for the aFMC significantly deviates from two seconds (2.34 \pm 0.13 seconds), as would be expected for a purely target/response-locked activation suggested by the onset latency difference of $1.84 \pm 0.20$ seconds. Since the absolute peak latency at CTI2000 exceeds the trial-border of six seconds $(7.50$ seconds), a possible explanation is that BOLD overlap from the following trial causes this distortion. Indeed, compensating for intertrial overlap by subtracting the null event time course results in a peak latency difference of exactly two seconds. Interestingly, when subtracting null events for brain areas in which absolute peak latencies did not considerably exceed the trialborder of six seconds (VC, pIFS, and MC), no significant distortion of peak latency difference was evident.

\section{PreSMA}

The observation that the peak latency difference $(1.81 \pm$ 0.26 seconds) exceeds the onset latency difference (0.87 \pm 0.36 seconds) suggests that PreSMA activation at CTI2000 (like VC or IFS) does not reflect the impact of a single focal event but shows additional activation due to subsequent processing. The standard error for both latency differences is almost twice as much as for the other brain areas we investigated. This result fits nicely

Figure 4. Grand-averages of trial-averaged time courses for the three exemplary frontal brain areas involved in task switching. Black curves marked by squares = CTI100, black curves marked by crosses $=$ CTI2000, gray curves marked by triangles $=$ null event. The temporal resolution of $125 \mathrm{msec}$. was obtained by linear interpolation. The left pIFS exhibits a cue-locked plus target-locked activation (with the same onset latencies for CTI100 and CTI2000, paralleled by a shifted peak latency for CTI2000). The PreSMA shows an activation that starts within the 2000 msec preparation interval (with a shifted onset latency of 0.87 seconds). The aFMC is activated on a purely target-locked basis (with a shifted onset latency and shifted peak latency for CTI2000 of about two seconds). 
with the fact that PreSMA is not tightly locked to some external event (as compared to VC or pIFS, which are directly triggered by cue presentation), but reflects a self-paced process that can not be timed as precisely as some externally-driven process.

\section{DISCUSSION}

We have proposed a method that had been claimed to be useful for the event-related analysis of an experimental design (cued task switching), which could not be optimized according to the constraints posed by existing methods based on linear decomposition of BOLD components $(4,7,12,13)$. In particular, event order could not be counterbalanced for logical reasons and the free choice of event spacings was restricted.

Our approach relied on the quantification of onset latencies and peak latencies being directly extracted from preprocessed trial-averaged fMRI time courses. The method yields yes-no decisions about the presence or absence of BOLD components associated with different temporally-defined event types. Since it includes the quantification of onset latencies (independently from peak latencies), additional information can be gained about the exact timing of BOLD components that arise within the two second preparation interval (see the exemplary result for PreSMA). The proposed method focused on determining the mapping between the temporal event structure and temporally-defined characteristics of the trial-related BOLD response. BOLD amplitudes were not considered, which implies that information about the relative quantitative contribution associated with different event types cannot, in principle, be recovered.

In principle, there are two alternative approaches to obtain estimates of temporally-defined features of the BOLD response, such as onset latencies and peak latencies. One is to fit some prespecified model function comprising a set of parameters that can be interpreted as indicators of the characteristics of interest (11,20,33-35). However, estimating onset latencies and peak latencies independently from each other cannot easily be achieved even with sophisticated nonlinear fitting procedures, due to tradeoffs between estimates of onset latency and peak latency (33).

Therefore, we implemented another approach based on preprocessed trial-averaged time courses $(36,37)$. Here, the onset latencies and peak latencies were directly extracted from BOLD time courses that have been selectively averaged across experimental conditions. The advantage of this procedure lies in its simplicity and its transparency with respect to the original data.

The statistical evaluation of onset latencies and peak latencies required the application of a nonstandard procedure. Usually, the calculation of group statistics relies on a distribution of values taken from individual subjects. However, reliably identifying onsets and peaks in trialaveraged time courses of single subjects turned out to be difficult. Due to the low signal-to-noise ratio, local minima are a substantial problem in particular with respect to BOLD onsets (37). Since our method relied on detecting small latency differences between conditions, smoothing the time courses with a broad filter was considered to be an insufficient solution, because it implies a reduced sensitivity. Therefore, we implemented another procedure already proven and tested in ERP research (30-32) - jackknife resampling - to improve the signal-to-noise ratio without distorting the temporal structure of the original time course.

\section{Validation}

Internal validation for our method is provided by the temporal patterns observed for both the visual cortex and the motor cortex. The results agreed quite well with the predictions from a simple linear model, suggesting that distorting effects due to nonlinear BOLD superposition and/or due to the inflection point criterion used for determining BOLD onsets do not pose a major problem-at least regarding the particular design we analyzed. However, peak latencies are subject to some distortion due to BOLD overlap from the subsequent trial when absolute peak-latencies exceed the trial border of six seconds (see exemplary results for the aFMC).

External validation for the results we obtained for task-switching-related brain areas comes from another task switching study conducted by Brass and von Cramon (38). In addition to basic cue-target trials, they included trials in which only a task cue was presented. They found a similar dissociation of brain areas with respect to cue-related and target-related activation; the PreSMA and the posterior IFS were activated in the cue-only condition, compared to the aFMC, which was only activated in the cue plus target condition. Extending the results obtained by Brass and von Cramon (38) that relied on the general linear model (GLM) framework, our method could detect subtle differences between the temporal patterns of the PreSMA and the posterior IFS.

Another important difference to experimental designs like that proposed by Brass and von Cramon (38) concerns assumptions about additive cognitive factors. Isolating cue-related activation by omitting the target stimulus relies strongly on the prerequisite condition that omitting the target does not add a new cognitive process (e.g., related to disconfirming the predicted target occurrence). Furthermore, omitting the task cue to isolate target-related activation is not logically possible. The approach we proposed is less likely to induce nonadditive processes, since the basic event structure is not manipulated.

One important aspect of this article was to demonstrate how temporal aspects of the BOLD signal can be analyzed driven purely by the data, on the basis of preprocessed trial-averaged time courses. The jackknife procedure allowed for evaluating features of the BOLD response that could easily be defined by general constraints, thus providing a complement to methods which are based on fitting some prespecified model function $(14,33)$. Probably the most commonly used analytical framework relies on the GLM including a set of additional basis functions for capturing variations in the shape of the BOLD response $(20,39)$. Considering the temporal derivative provides information about the delay of the BOLD response, but does not differentiate between effects due to variations in either onset laten- 
cies or peak latencies. As exemplified in this article, this information is not sufficient when more fine-grained analyses of the temporal structure of the event-related BOLD response are required.

\section{ACKNOWLEDGMENTS}

We thank Orit Rubin, Birte Forstmann, Jane Neumann, and two anonymous reviewers for helpful comments on the manuscript.

\section{REFERENCES}

1. Boynton GM, Engel SA, Glover GH, Heeger DJ. Linear systems analysis of functional magnetic resonance imaging in human. J Neurosci 1996;16:4207-4221.

2. Buckner RL, Bandettini PA, O'Craven KM, et al. Detection of cortical activation during averaged single trials of a cognitive task using functional magnetic resonance imaging. Proc Natl Acad Sci USA 1996;93:14878-14883.

3. Rosen BR, Buckner RL, Dale AM. Event-related function MRI: past, present, and future. Proc Natl Acad Sci USA 1998;95:773-780.

4. Burock MA, Buckner RL, Woldorff MG, Rosen BR, Dale AM. Randomized event-related experimental designs allow for extremely rapid presentation rates using functional MRI. Neuroreport 1998; 9:3735-3739.

5. Dale AM. Optimal experimental design for event-related fMRI. Hum Brain Mapp 199;8:109-114.

6. Josephs O, Henson RNA. Event-related functional magnetic resonance imaging: modelling, inference and optimisation. Phil Trans R Soc Lond B 1999;354:1215-1228.

7. Bandettini PA, Cox RW. Event-related fMRI contrast when using constant interstimulus interval: theory and experiment. Magn Reson Med 2000;43:540-548.

8. Dale AM, Buckner RL. Selective averaging of rapidly presented individual trials using fMRI. Hum Brain Mapp 1997;5:329-340.

9. Friston KJ, Zarahn E, Josephs O, Henson RNA, Dale AM. Stochastic designs in event-related fMRI. Neuroimage 1999;9:416-429.

10. Pollmann S, Dove A, von Cramon DY, Wiggins CJ. Event-related fMRI: Comparison of conditions with varying BOLD overlap. Hum Brain Mapp 2000;9:26-37.

11. Josephs O, Turner R, Friston K. Event-related fMRI. Hum Brain Mapp 1997;5:243-248.

12. Toni I, Thoenissen D, Zilles K. Movement preparation and motor intention. Neuroimage 2001;14:110-117.

13. Glover GH. Deconvolution of impulse response in event-related BOLD fMRI. Neuroimage 1999;9:416-429.

14. Kruggel F, Zysset S, von Cramon DY. Modelling multiple hemodynamic responses in event-related functional MRI experiments. In: Gedeon F, Wong P, Halgamuge S, Kasabov, N, Nauck D, Fukushima K, editors. 6th International Conference on Neural Information Processing (ICONAP 99). Piscataway, NJ: IEEE Press; 1999. p 903-909.

15. Meiran N. Reconfiguration of processing mode prior to task performance. J Exp Psychol Learn Mem Cogn 1996;22:1423-1442.

16. Meiran N, Chorev Z, Sapir A. Component processes in task switching. Cognit Psychol 2000;41:211-253.

17. Oldfield RC. The assessment and analysis of handedness: The Edinburgh Inventory. Neuropsychologia 1971;9:97-113.
18. Lohmann G, Mueller K, Bosch V, et al. Lipsia: a new software system for the evaluation of functional magnetic resonance images of the human brain. Comput Med Imaging Graph 2001;25:449457.

19. Talairach P, Tournoux J. A stereotactic coplanar atlas of the human brain. Stuttgart, Germany: Thieme; 1988. 122 p.

20. Friston KJ, Fletcher P, Josephs O, Holmes A, Rugg MD, Turner R. Event-related fMRI: characterizing differential responses. Neuroimage 1998;730-740.

21. Hopfinger JB, Buechel C, Holmes AP, Friston KJ. A study of analysis parameters that influence the sensitivity of event-related analyses. Neuroimage 2000;11:326-333.

22. Vazques AL, Noll DC. Nonlinear aspects of the BOLD response in functional MRI. Neuroimage 1998;7:108-118.

23. Friston KJ, Josephs O, Rees G, Turner R. Nonlinear event-related responses in fMRI. Magn Reson Med 1998;37:41-52.

24. Huettel SA, McCarthy G. Evidence for a refractory period in the hemodynamic response to visual stimuli as measured by fMRI. Neuroimage 2000;11:547-553.

25. Buckner RL, Koutstaal W, Schacter DL, Dale AM, Rotte MR, Rosen BR. Functional-anatomic study of episodic retrieval: II. Selective averaging of event-related fMRI trials to test the retrieval success hypothesis. Neuroimage 1998;7:163-175.

26. Schacter DL, Buckner RL, Koutstaal W, Dale AM, Rosen BR. Late onset of anterior prefrontal activity during true and false recognition: an event-related fMRI study. Neuroimage 1997;6:259-269.

27. Raichle ME, MacLeod AM, Snyder AZ, Powers WJ, Gusnard DA, Shulman GL. A default mode of brain functioning. Proc Natl Acad Sci USA 2001;98:676-682.

28. Efron B. Nonparametric estimates of standard error: the jackknife, the bootstrap, and other methods. Biometrika 1981;68:589-599.

29. Miller RG. The jackknife: a review. Biometrika 1974;61:1-15.

30. Miller J, Patterson T, Ulrich R. Jackknife-based method for measuring LRP onset latency differences. Psychophysiology 1998;35: 99-115.

31. Mordkoff JT, Gianaros PJ. Detecting the onset of the lateralized readiness potential: A comparison of available methods and procedures. Psychophysiology 2000;37:347-360.

32. Ulrich R, Miller J. Using the Jackknife-based scoring method for measuring LRP onset effects in factorial designs. Psychophysiology 2001;38:816-827.

33. Henson RNA, Rugg MD. Effects of stimulus repetition on latency of the BOLD impulse response. Neuroimage 2001;13:683.

34. Kruggel F, von Cramon DY. Modelling the hemodynamic response in single-trial functional MRI experiments. Magn Reson Med 1999; 42:787-797.

35. Miezin FM, Macotta L, Ollinger JM, Petersen SE, Buckner RL. Characterizing the hemodynamic response: effects of presentation rate, sampling procedure, and the possibility of ordering brain activity based on relative timing. Neuroimage 2000;11:735-759.

36. Menon RS, Luknowsky DC, Gati JS. Mental chronometry using latency-resolved functional MRI. Proc Natl Acad Sci USA 1998;95: 10902-10907.

37. Neumann J, Lohmann G, Zysset S, von Cramon DY. Within-subject variability of BOLD response dynamics. Neuroimage 2003;19:773785 .

38. Brass M, von Cramon DY. The role of the frontal cortex in task preparation. Cereb Cortex 2002;12:908-914.

39. Henson RNA, Price C, Rugg MD, Turner R, Friston K. Detecting latency differences in event-related BOLD responses: application to words vs. nonwords, and initial vs. repeated face presentations. Neuroimage 2002;15:83-97. 